\title{
Fermi bubbles around the M31 galaxy
}

\author{
Maxim Pshirkov ${ }^{1,2,3, \star}$, Valeriy Vasiliev ${ }^{4, \star \star}$, and Konstantin Postnov ${ }^{1, \star \star \star}$ \\ ${ }^{1}$ Sternberg Astronomical Institute, Lomonosov Moscow State University, Universitetsky prospekt 13, \\ 119992, Moscow, Russia \\ ${ }^{2}$ Institute for Nuclear Research of the Russian Academy of Sciences, 117312, Moscow, Russia \\ ${ }^{3}$ Pushchino Radio Astronomy Observatory, 142290 Pushchino, Russia \\ ${ }^{4}$ IMPRS Max Planck Institute for Astronomy,D-69117, Heidelberg, Germany
}

\begin{abstract}
We have searched for an extended high-energy gamma-ray emission around the nearby giant spiral Andromeda galaxy M31 using almost 7 years of Fermi LAT data at energies above $300 \mathrm{MeV}$. The presence of a diffuse gamma-ray halo with total photon flux $2.6 \pm 0.6 \times 10^{-9} \mathrm{~cm}^{-2} \mathrm{~s}^{-1}$, corresponding to a luminosity $(0.3-100 \mathrm{GeV})$ of $(3.2 \pm 0.6) \times 10^{38}$ $\mathrm{erg} \mathrm{s}^{-1}$ (for a distance of $780 \mathrm{kpc}$ ) was found at a $5.3 \sigma$ confidence level. The best-fit halo template corresponds to two 6-7.5 kpc bubbles symmetrically located perpendicular to the M31 galactic disc, similar to the 'Fermi bubbles' found around the Milky Way centre.
\end{abstract}

\section{Introduction}

There are several observational manifestations of extended baryonic coronae around spiral galaxies: soft diffuse X-ray emission extending up to several ten kpc from the central galaxy [1], absorption in O VII line [2,3], distortions in the shape of gas clouds [4] and stripping of gas in the satellite galaxies by the ram-pressure of the halo gas [5], see [6] and references therein for a review. Such a hot halo around the Milky Way is established by several different methods [7].

Spiral galaxies can also be surrounded by extended cosmic-ray $(\mathrm{CR})$ halos $[8,9]$ : if there were strong enough magnetic fields (10-100 nG) far away from central regions of the Galaxy, CRs, generated in galaxies, would remain in the magnetized Galactic halo for a cosmological time. These CRs would interact with tenuous $\left(\sim 10^{-4} \mathrm{~cm}^{-3}\right)$ hot plasma producing gamma-rays via pionic channel, the gamma-ray luminosity of such a halo could be around $10^{39} \mathrm{erg} \mathrm{s}^{-1}$ at energies above $100 \mathrm{MeV}$ [9]. The size and shape of the halo cannot be established from first principles and depend on the propagation properties of CRs, its predicted size falls in the 20-40 kpc range[9], at smaller scales $(\sim 10 \mathrm{kpc})$, it can be non-uniform as evidenced by the 'Fermi bubbles' (FB) in the Galaxy [10, 11].

It is almost impossible to disentangle such a halo in our Galaxy from the truly extragalactic component. However, they can be searched for around other spiral galaxies. The most natural target is the nearest M31 (Andromeda) galaxy. One can expect the halo size of order of several degrees that can be detected from the Earth-M31 distance of $>700 \mathrm{kpc}$. The presence of a hot gas around M31, which

\footnotetext{
^e-mail: pshirkov@sai.msu.ru

$\star \star$ e-mail: vasilyev@mpia.de

$\star \star \star$ e-mail: kp@sai.msu.ru
} 


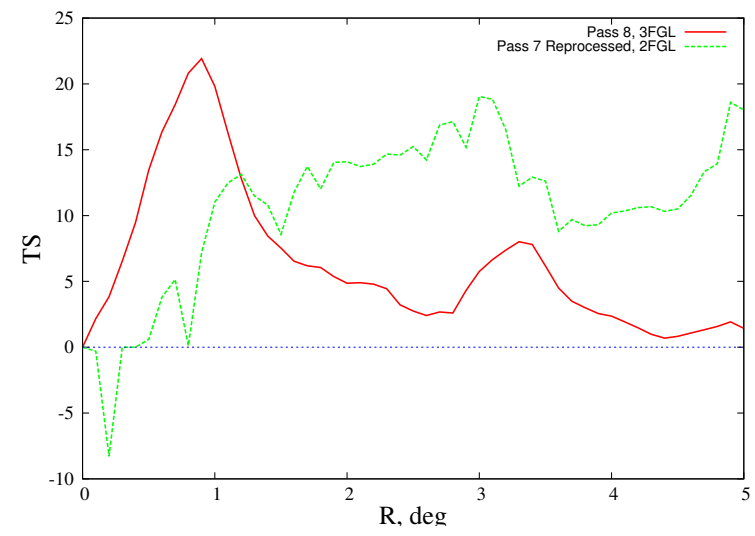

Figure 1. $T S$ ( $\left.R_{\text {halo }}\right)$ curves: an earlier version (65 month of data, Pass7 Reprocessed events and 2FGL catalogue) is shown for comparison. The $T S\left(R_{\text {halo }}\right)$ curve is much smoother when the latest version of event reconstruction and the 3FGL source catalogue are used.

is essential for the gamma-ray emission from the CR halo, was recently demonstrated by the discovery of certain absorption features in UV-spectrum of quasars projected on the sky close to the galaxy $[12,13]$ and distortions in the observed CMB spectrum in the vicinity of M31 due to interference from the halo gas [14].

\section{Data and data analysis}

In our analysis we have used 83 months of Fermi LAT data collected since 2008 Aug 04 until 2015 Jul 06. We have selected events that belong to the "SOURCE" class, PASS8_V2 reconstruction and $\mathrm{v} 10 \mathrm{r} 0 \mathrm{p} 5^{1}$ version of the Fermi science tools was used. Only events with energies larger than 300 $\mathrm{MeV}$ were selected, because at lower energies the Fermi LAT point spread function (PSF) quickly deteriorates. Usual event quality cut, namely that the zenith angle should be less than $100^{\circ}$ (which is sufficient at these energies) has been imposed. For further details of analysis, see [15].

\section{Results}

\subsection{Uniform circle template}

Firstly, we searched for diffuse gamma-ray emission from the M31 galaxy. The galaxy was modelled in two different ways: as a point-like source or as an extended object (the IR-based templat, IRAS). The extended template for the M31 galaxy fits the data considerably better than the simple point-like source $\left(T S_{\mathrm{ext}}=79, T S_{\mathrm{ps}}=62.3\right)$. The galaxy has a soft spectrum with photon index $\Gamma=2.40 \pm 0.12$ and the flux $F=(2.6 \pm 0.4) \times 10^{-9} \mathrm{ph} \mathrm{cm}^{-2} \mathrm{~s}^{-1}$ in the $0.3-100 \mathrm{GeV}$ energy range. The spectrum is even softer if the galaxy is modelled as a point-like source: $\Gamma=2.64 \pm 0.15$ with the photon flux $F=(1.9 \pm 0.3) \times 10^{-9} \mathrm{ph} \mathrm{cm}^{-2} \mathrm{~s}^{-1}$.

The results of fitting with additional halo component are presented in Fig. 1. The fit quality improvement can be easily seen. The highest statistical significance $T S=22$ was obtained for a

\footnotetext{
${ }^{1}$ http://fermi.gsfc.nasa.gov/ssc/data/analysis/software/
} 


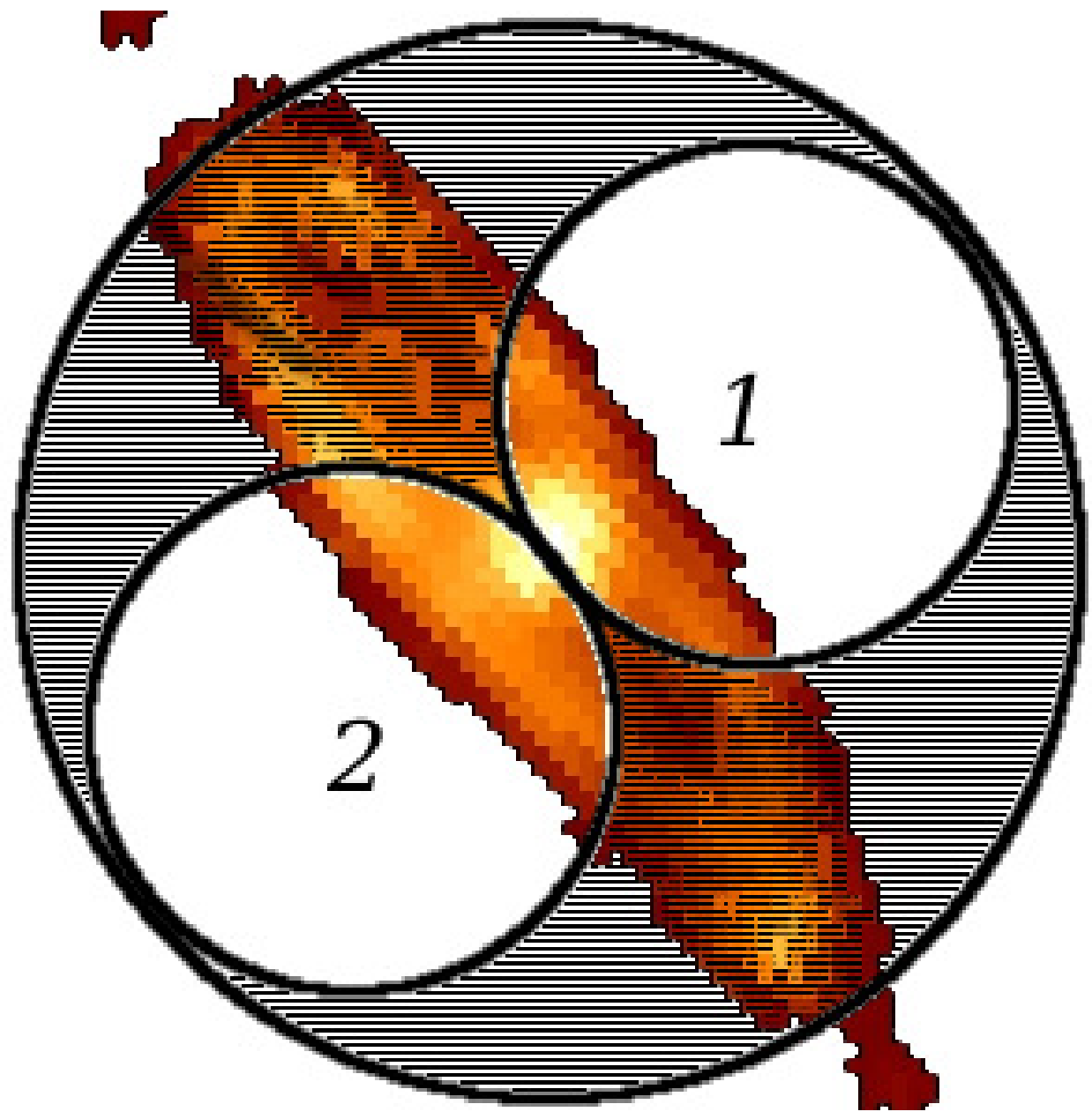

Figure 2. Model templates: IRAS template, $0.9^{\circ}$ uniform circle, (1) and (2) $-0.45^{\circ}$ bubbles perpendicular to the galactic plane, and region complementary to two bubbles (the shaded area).

halo with radius $R_{\text {halo }}=0.9^{\circ}$, corresponding to a linear size of $\sim 12 \mathrm{kpc}$. The photon flux from the extended halo and the $0.3-100 \mathrm{GeV}$ luminosity obtained from the fit are $\sim(3.2 \pm 1.0) \times 10^{-9} \mathrm{~cm}^{-2} \mathrm{~s}^{-1}$ and $(4.0 \pm 1.5) \times 10^{38} \mathrm{erg} \mathrm{s}^{-1}$, respectively, adopting the distance $d=780 \mathrm{kpc}$. The spectral index is found to be rather soft: $\Gamma=2.30 \pm 0.12$. A marginal improvement $(T S \sim 8)$ could be also achieved by adding a $3^{\circ}$ halo $(\sim 35 \mathrm{kpc})$.

\subsection{Bubble template}

Despite low statistics (600 - 700 photons), some information about the possible nature of the found extended emission could be inferred from its morphology. The first natural option is that the emission has hadronic origin - the cosmic rays are interacting with the diffuse medium, producing gamma-rays. 


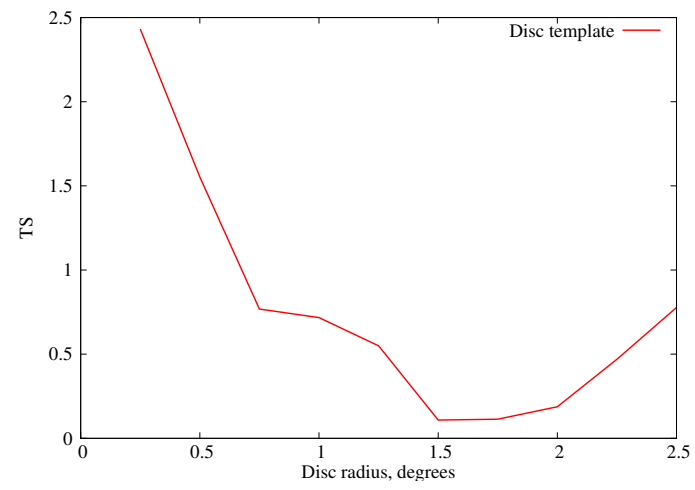

Figure 3. $T S$ curve for an elliptic template with aspect ratio $a / b=0.22$, representing addition of the HI disc-like emission. Inclusion of these templates does not improve the fit quality.

In this case the emission brightness traces the concentration of targets, i.e., the gas density. Most of the gas mass of the M31 galaxy resides in a flat HI disc (see, e.g [16]) and the expected template should naturally have the shape of an ellipse with aspect ratio $b / a=\cos i \sim 0.22$, where $a, b$ are the semi-major and semi-minor axes, respectively, and $i=77^{\circ}$ is the inclination angle. The results are presented in Fig. 3: addition of such a disc-like component does not improve the fit quality. This implies that the hadronic origin of the extended halo due to the cosmic rays interactions with matter is strongly disfavoured.

The Fermi bubbles are almost circular regions of $\sim 6 \mathrm{kpc}$ radius located above and below the Galactic centre, their total luminosity is equal to $L_{\mathrm{FB}} \sim 4 \times 10^{37} \mathrm{erg} \mathrm{s}^{-1}$. They are believed to stem from the past activity of the central region of the Galaxy, either from the central supermassive black hole (SMBH) or central star-formation burst. We decided to test whether a 'FB-like' morphology can fit the data better than the simple uniform disc template. First of all, we performed fits varying the bubble radius from 0.1 to 0.75 degrees. For each bubble radius, four different models were fitted (see Fig. 2). The results are shown in Fig. 4. There is a clear maximum for the FB $1+2$ template with $0.45-0.55^{\circ}$ radius of the bubbles (corresponding to the linear size 6-7.5 kpc), with $T S=28.2$. The rest of the $0.9^{\circ}$ circle $\left(\mathrm{FB}_{\text {compl }}\right.$ template) contributes very little. The parameters of the best-fit model are listed in Table 1. Finally, we have searched for a possible ellipticity of the bubbles. The major semi-axes of both ellipses normal to the disc plane were fixed at $a=0.45^{\circ}$, while the aspect ratio $b / a$ was allowed to vary in the 0.1-0.9 range. Fig. 5 shows that although the signal is concentrated around the major semi-axes, it is not completely contained within this narrow jet-like region.

\section{Discussion and conclusions}

From almost 7 years of the Fermi-LAT observations, we find that the data suggest the presence of a spatially extended diffuse gamma-ray excess around M31. The best-fit morphology of the diffuse emission closely resembles the Fermi bubbles in the Milky Way. The best fit gave $~ 5.2 \sigma$ significance for two $0.45^{\circ}(6.5 \mathrm{kpc})$ bubbles with a photon flux of $\sim(2.6 \pm 0.6) \times 10^{-9} \mathrm{~cm}^{-2} \mathrm{~s}^{-1}$ and a luminosity of $(3.2 \pm 0.6) \times 10^{38} \mathrm{erg} \mathrm{s}^{-1}$ in the energy range $0.3-100 \mathrm{GeV}$. These parameters are fairly close to those of the Fermi bubbles in the Milky Way, though the luminosity of the latter ones is about one order of magnitude lower. The difference in the luminosity can be explained by the presence of a much more 


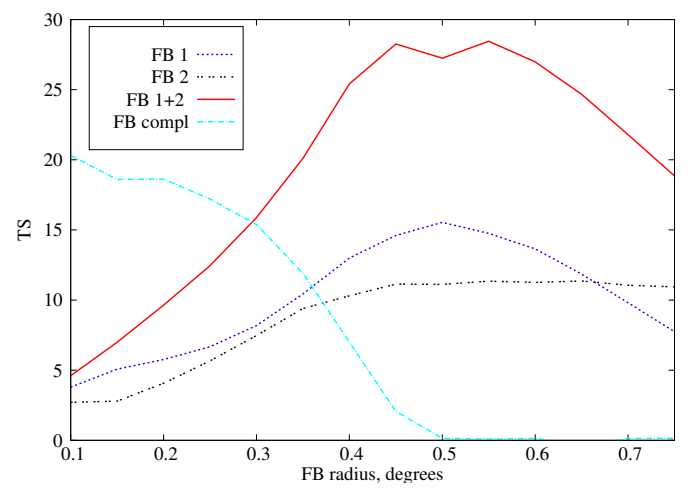

Figure 4. TS curves for different templates. Most of the signal comes from the region corresponding to the FB $1+2$ template.

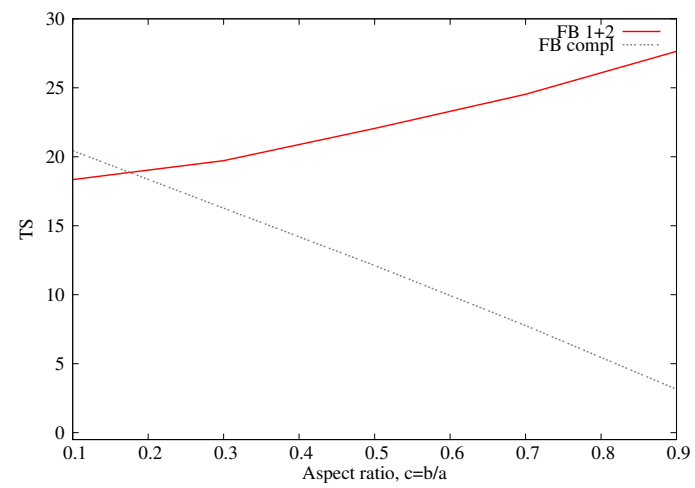

Figure 5. TS curve for elliptic FB templates with different aspect ratio $b / a$. The semi-major axis is fixed at $a=0.45^{\circ}$. The complementary template is defined as above (see Fig. 2)

Table 1. Best-fit parameters for IRAS + different halo templates from Fig. 2. Column 1: model template, column 2: $T S$ value, column 3: integral flux in the $0.3-100 \mathrm{GeV}$ range, column 4: photon spectral index $\Gamma$. For comparison, best-fit parameters for uniform $0.9^{\circ}$ template are also presented

\begin{tabular}{|c|c|c|c|}
\hline model & $T S$ & $\begin{array}{c}F_{300}, 10^{-9} \\
\left(\mathrm{~cm}^{-2} \mathrm{~s}^{-1}\right)\end{array}$ & $\Gamma$ \\
\hline \hline IRAS only & - & $2.9 \pm 0.4$ & $2.4 \pm 0.1$ \\
FB 1 & 14.6 & $1.4 \pm 0.5$ & $2.5 \pm 0.3$ \\
FB 2 & 11.1 & $0.72 \pm 0.35$ & $2.0 \pm 0.2$ \\
FB 1+2 & 28.2 & $2.6 \pm 0.6$ & $2.3 \pm 0.1$ \\
FB $_{\text {compl }}$ & 2.0 & $1.1 \pm 0.9$ & $2.5 \pm 0.4$ \\
\hline $0.9^{\circ}$ circle & 22.0 & $3.2 \pm 1.0$ & $2.3 \pm 0.1$ \\
\hline
\end{tabular}


massive SMBH in the M31 centre. Our findings suggest that the FB phenomenon can be widespread in giant spiral galaxies.

\section{Acknowledgements}

The work was supported by the Grant of the President of Russian Federation MK-4167.2015.2 and RFBR grant 14-02-00657.

\section{References}

[1] J.T. Li, Z. Li, Q.D. Wang, J.A. Irwin, J. Rossa, MNRAS390, 59 (2008), 0807.3587

[2] Q.D. Wang, Y. Yao, T.M. Tripp, T.T. Fang, W. Cui, F. Nicastro, S. Mathur, R.J. Williams, L. Song, R. Croft, ApJ635, 386 (2005), astro-ph/0508661

[3] J.N. Bregman, E.J. Lloyd-Davies, ApJ669, 990 (2007), 0707 . 1699

[4] T. Westmeier, R. Braun, D. Thilker, A\&A436, 101 (2005), astro-ph/0503246

[5] L. Blitz, T. Robishaw, ApJ541, 675 (2000), astro-ph/0001142

[6] M.E. Putman, J.E.G. Peek, M.R. Joung, ARA\&A50, 491 (2012), 1207.4837

[7] M.J. Miller, J.N. Bregman, ApJ770, 118 (2013), 1305. 2430

[8] F. De Paolis, G. Ingrosso, P. Jetzer, M. Roncadelli, ApJ510, L103 (1999), astro-ph/9901033

[9] R. Feldmann, D. Hooper, N.Y. Gnedin, ApJ763, 21 (2013), 1205.0249

[10] M. Su, T.R. Slatyer, D.P. Finkbeiner, ApJ724, 1044 (2010), 1005.5480

[11] M. Ackermann et al. (Fermi-LAT), Astrophys. J. 793, 64 (2014), 1407.7905

[12] S.M. Rao, G. Sardane, D.A. Turnshek, D. Thilker, R. Walterbos, D. Vanden Berk, D.G. York, MNRAS432, 866 (2013), 1302.7026

[13] N. Lehner, J.C. Howk, B.P. Wakker, ApJ804, 79 (2015), 1404.6540

[14] F. De Paolis, V.G. Gurzadyan, A.A. Nucita, G. Ingrosso, A.L. Kashin, H.G. Khachatryan, S. Mirzoyan, E. Poghosian, P. Jetzer, A. Qadir et al., A\&A565, L3 (2014), 1404 . 4162

[15] M.S. Pshirkov, V.V. Vasiliev, K.A. Postnov, MNRAS459, L76 (2016), 1603.07245

[16] T.R. Cram, M.S. Roberts, R.N. Whitehurst, A\&AS40, 215 (1980) 